\title{
The Relationship between Helicobacter Pylori Virulence Factors and Gastric Carcinoma
}

\author{
Mohamed Shees Ahmed", Mohamed Amer Afifi", Hany Mostafa Abu-Shukka*, \\ Ragaae Mohamede Fath-Elbab**, Raafat Zaher Abdel Rahman**, Ahmed Fkirin ** \\ * Microbiology and Immunology Department, Faculty of Medicine, AL-Azhar University, \\ Cairo, Egypt. **, Military Medical Academy, Cairo, Egypt. \\ Corresponding author: Ahmed Fkirin, E-mail: Ahmed_fk@yahoo.com
}

\begin{abstract}
Background: Helicobacter pylori is a gram-negative microaerophilic bacterium found usually in the stomach and use several mechanisms to survive in the stomach lumen. The presence of these bacteria in the stomach can lead to gastritis and reduction in stomach acid production. Aim of the work: this study aimed to investigate the seroprevalence of six highly immunogenic virulence factors (Cag A, Vac A, Gro EL, gGT, Hcp C and Ure A) in patients with different gastric histology in a high-risk population of gastric cancer and explore the relationship between $H$. pylori virulence factors and gastric carcinoma. Methods: this study included 100 patients: 25 patients were diagnosed histopathologically as gastric cancer (Group I) and 75 patients were diagnosed endoscopically as mild gastritis (Group II). Their samples were collected from Military Armed Hospitals. Noninvasive serologic test was performed to detect immune responses to $H$. pylori by stool antigen test, ELISA and by Recomoline $H$. pylori test.

Results: on comparing results of gastric carcinoma and chronic gastritis by Recomoline H. pylori test, it was found that Cag A was detected in $88 \%$ (22/25) in group I. While, in group II it was $57.33 \%$ (43/75) of cases and Vac A was detected in $80 \%(20 / 25)$ in group I while, it was in group II in 44\% (33/75) of cases. Gro EL was detected in $72 \%$ (18/25) in group I while; it was 49.33\% (37/75) group II and Urea A was 52\% (13/25) in group I while, it was $52 \%(39 / 75)$ in group II. Hcp c was 56\% (14/25) in group I while, it was 40\% (30/75) in group II and gGT was $48 \%(12 / 25)$ in group I while, it was $34.67 \%(26 / 75)$ in group II.

Conclusion: in this study, a statistically significant association was found between Cag A, Vac A and Gro EL antigen in the studied groups of patients. However, no such statistically significant association was found between Urea A, Hcp c and gGT antigen. (P value > 0.05). Thus, Cag A, Vac A and Gro EL antigen proved to be virulence markers for gastric carcinoma group.
\end{abstract}

Keywords: Helicobacter pylori; Recomoline H. pylori test.

\section{INTRODUCTION}

In $1994, H$. pylori was recognized as a type I carcinogen and it was considered as the most common etiologic agent of infection-related cancers, which represented $5.5 \%$ of the global cancer burden. In 2005, Marshall and Warren were awarded the Nobel Prize of Medicine for their discovery of this bacterium and its role in peptic ulcer disease ${ }^{[1]}$. In areas with high incidence of gastric cancer, population-based screening and treatment measures were discussed to reduce the incidence of gastric cancer ${ }^{[2]}$. Japan has recently revised its guidelines and now recommends screening for $H$. pylori infection and treatment of all the infected individuals ${ }^{[3]}$.

The most well-known H. pylori risk marker was the cytotoxin-associated antigen (Cag A), a component of the pathogenicity island, which was present in approximately $60 \%$ of $\mathrm{H}$. pylori strains in the United States ${ }^{[4]}$. Cag A contributes to alteration of myriad signaling transduction, which affects host cell physiology with disruption of intercellular junctions, loss of cell polarity, promotion of inflammation, dysregulation of cellular apoptosis and proliferation. Vac A inducts cytoplasmic vacuolation, apoptosis and immune suppression ${ }^{[5]}$. Many testing methods are available for detection of $H$. pylori. Invasive methods use endoscopy as the vehicle to obtain tissue for histology, noninvasive tests are recommended for first-line assessment of $H$. pylori infection. Here, the $\left[{ }^{13} \mathrm{C}\right]$ urea breaths test (UBT), serology and the stool antigen test offer high accuracy at relatively low cost. Culture is no longer considered necessary for confirmation of $H$. pylori infection, but cultured organisms can be tested for antibiotic resistance. Biopsies are also required for PCR analysis ${ }^{[6]}$.

\section{AIM OF THE WORK}

This study aimed to investigate the seroprevalence of six highly immunogenic virulence factors (Cag A, Vac A, Gro EL, gGT, Hcp C and Ure A) in patients with different gastric histology in a high-risk population of gastric cancer and explores the relationship between $H$. pylori virulence factors and gastric carcinoma.

\section{PATIENTS AND METHODS}

\section{Study design:}

This study was conducted between January 2015 and November 2016. Specimens were obtained from the Endoscopy Unit and Surgical Oncology Department, 
Military Armed Hospitals. The microbiological work was performed at the Medical Microbiology and Immunology Department, The Armed Forces of the Central Laboratory for Medical Research and the Blood Bank. One hundred patients were included in this study and categorized according to their findings into 2 groups:

Group I: Comprised 25 patients who had gastric cancer subjected to gastroduodenoscopy at the Endoscopy Unit or to gastric surgery (whether subtotal or total gastrectomy) at the Surgical Oncology Department. The presence of gastric cancer was confirmed by the Surgical Pathology Department.

Group II: comprised 75 patients who had persistent symptoms suggestive of gastritis and subjected to gastroduodenoscopy at the Endoscopy unit.

Personal history (including age, sex, smoking, etc.), patient's complaints, family history, past history [specially any previous gastric complaint, gastric surgery, treatment with proton pump inhibitors (PPI) or any specific treatment given for $H$. pylori eradication in the past] as well as all endoscopic findings were recorded for each patient.

\section{Exclusion Criteria:}

Patients were excluded from the study if They suffered from other primary malignancies or inflammatory diseases, they had received any antimicrobial treatment or PPI for the previous 3 months and they had received chemotherapy or radiation therapy prior to endoscopy or surgery.

Prior to their enrollment in the present study, an informed consent was obtained from each patient.

\section{Serum sample collection:}

Before endoscopy, two samples up to $10 \mathrm{ml}$ of whole blood were collected. Blood samples could stand for 30-40 min and serum separation were accomplished by centrifugation at $965 \mathrm{~g}$ for $15 \mathrm{~min}$. The supernatant serum was recovered and stored at $-80^{\circ} \mathrm{C}$ until analysis. One serum sample was used for ELISA test and the other one was processed and used for Recomoline test.

\section{Endoscopy and histological evaluation:}

At endoscopy, group I subjects were evaluated for the presence of any gastric tumors, while group II subjects were evaluated for the presence of gastritis.

\section{Immunological examination:}

Stool antigen test:

The stool antigen test detects antigen presence in the feces that indicates active $\mathrm{H}$. pylori infection. It can be also used to monitor the effectiveness of treatment and the recurrence of the infection.

ELISA:

Serology is one of the most widely employed methods for screening larger populations and several test systems have been developed. They are cheap and easy to perform but either cannot discriminate between type I (highly virulent) and type II strains (reduced virulence) or suffer from a lack of sensitivity [7].

Recomline immunoassay:

H. pylori Recomline serology was developed to detect antibody levels directed against six $H$. pylori immunogenic proteins (Cag A, Vac A, Gro EL, gGT, Hcp $\mathrm{C}$ and Ure A). In contrast to ELISA, the test principle allows the identification of specific antibodies against various antigens of $H$. pylori through the separate application of different single antigens. Highly purified recombinant $H$. pylori antigens were fixed on nitrocellulose membrane strips ${ }^{[6]}$.

Since some $H$. pylori strains have been shown to lead to more severe clinical outcomes than others, antigens like Vac A and Cag A were included. These proteins are linked to the development of ulcers or premalignant changes and gastric cancer. Thus, the assay might be able to distinguish between a type I (more virulent) strain infection if Cag A and/or Vac A are positive and a type II strain infection if only one or more of the other antigens (Gro EL, Ure A, Hcp C and gGT) are positive ${ }^{[5]}$.

This study was done according to the ethical board of Al-Azhar University. Statistical analysis:

Data were analyzed using Statistical Program for Social Science (SPSS) version 15.0.Quantitative data were expressed as mean \pm standard deviation (SD). Qualitative data were expressed as frequency and percentage.

The following tests were done:

Chi-square test: was used when comparing between non-parametric data.

A one-way analysis of variance (ANOVA): when comparing between more than two means.

Post Hoc test: was used for multiple comparisons between different variables. Probability (P-value)

- P-value $<0.05$ was considered significant.

- P-value $<0.001$ was considered as highly significant.

- P-value >0.05 was considered insignificant. 


\section{RESULTS}

Table 1: age distribution among cancer cases (Group I) and gastritis cases (Group II)

\begin{tabular}{|c|c|c|c|c|c|c|c|}
\hline \multirow{2}{*}{ Groups } & \multicolumn{5}{|r|}{$\overline{\text { Age }}$} & \multicolumn{2}{|r|}{ T-test } \\
\hline & & Range & Mean & \pm & SD & $\mathbf{t}$ & P-value \\
\hline Group I & 32 & $\begin{array}{l}-64 \\
-\quad 64\end{array}$ & 53.400 & \pm & 7.687 & \multirow{2}{*}{5.084} & \multirow{2}{*}{$<0.001 *$} \\
\hline Group II & 18 & $\begin{array}{l}-70 \\
\end{array}$ & 40.733 & \pm & 16.981 & & \\
\hline
\end{tabular}

Table 1 showed age ranged from 32-64 years with mean $53.400 \pm 7.687$ in gastric cancer patients and ranged from 18-70 years with mean $40.733 \pm 16.981$ in gastritis patients with a statistically significant difference between the gastric carcinoma patients and the gastritis patients, age distribution was higher among gastric carcinoma patients (P value $<0.001)$.

Table 2: sex distribution of the studied groups

\begin{tabular}{|c|c|c|c|c|c|c|}
\hline & \multirow[t]{2}{*}{ Sex } & \multicolumn{3}{|r|}{ Groups } & \multicolumn{2}{|c|}{ Chi-square } \\
\hline & & Group I & Group II & Total & $\overline{X^{2}}$ & P-value \\
\hline \multirow[t]{2}{*}{ Female } & $\mathbf{N}$ & 9 & 51 & 60 & \multirow[t]{6}{*}{7.901} & \multirow[t]{6}{*}{$0.005 *$} \\
\hline & $\%$ & $36.0 \%$ & $68.0 \%$ & $60.0 \%$ & & \\
\hline \multirow[t]{2}{*}{ Male } & $\mathbf{N}$ & 16 & 24 & 40 & & \\
\hline & $\%$ & $64.0 \%$ & $32.0 \%$ & $40.0 \%$ & & \\
\hline \multirow[t]{2}{*}{ Total } & $\mathbf{N}$ & 25 & 75 & 100 & & \\
\hline & $\%$ & $100.0 \%$ & $100.0 \%$ & $100.0 \%$ & & \\
\hline
\end{tabular}

Table 2 showed among the studied groups, there were 40 males (16 in group I and 24 in group II) and 60 females ( 9 in group I and 51 in group II). The gender distribution showed statistically significant difference between the 2 studied groups.

Table 3: smoking habits of the studied groups

\begin{tabular}{|c|c|c|c|c|c|c|}
\hline \multirow{2}{*}{\multicolumn{2}{|c|}{\begin{tabular}{|l} 
Smoker \\
\end{tabular}}} & \multicolumn{3}{|r|}{ Groups } & \multicolumn{2}{|c|}{ Chi-square } \\
\hline & & Group I & Group II & Total & $\mathbf{X}^{2}$ & P-value \\
\hline \multirow[t]{2}{*}{ Non-smoker } & $\mathbf{N}$ & 15 & 56 & 71 & \multirow[t]{6}{*}{1.885} & \multirow[t]{6}{*}{0.170} \\
\hline & $\%$ & $60.0 \%$ & $74.7 \%$ & $71.0 \%$ & & \\
\hline \multirow[t]{2}{*}{ Smoker } & $\mathbf{N}$ & 10 & 19 & 29 & & \\
\hline & $\%$ & $40.0 \%$ & $25.3 \%$ & $29.0 \%$ & & \\
\hline \multirow[t]{2}{*}{ Total } & $\mathbf{N}$ & 25 & 75 & 100 & & \\
\hline & $\%$ & $100.0 \%$ & $100.0 \%$ & $100.0 \%$ & & \\
\hline
\end{tabular}

Table 3 showed that among the studied groups, there were 71 nonsmokers (15 in group I and 56 in group II) and 29 smokers (10 in group I and 19 in group II). The smoking distribution showed no statistically significant difference between the 2 studied groups.

Table 4: comparison between the six Helicobacter pylori virulence factors with gastric carcinoma and gastritis

\begin{tabular}{|c|c|c|c|c|c|c|c|c|c|}
\hline & \multicolumn{2}{|c|}{ Gastric carcinoma } & \multicolumn{2}{|c|}{ Chronic gastritis } & \multicolumn{2}{|c|}{ Total } & \multicolumn{2}{|c|}{ Chi-square } \\
\hline & & $\mathbf{N}$ & $\%$ & $\mathbf{N}$ & $\%$ & $\mathbf{N}$ & $\%$ & $\mathbf{X}^{2}$ & P-value \\
\hline & Cag A & 22 & $88 \%$ & 43 & $57.33 \%$ & 65 & 65 & 8.790 & $0.003 *$ \\
\hline & Vac A & 20 & $80 \%$ & 33 & $44.00 \%$ & 53 & 53 & 10.360 & $0.001 *$ \\
\hline & Gro EL & 18 & $72 \%$ & 37 & $49.33 \%$ & 55 & 55 & 4.021 & $0.045^{*}$ \\
\hline & Urea $A$ & 13 & $52 \%$ & 39 & $52.00 \%$ & 52 & 52 & 0.000 & 1.000 \\
\hline & Hсp c & 14 & $56 \%$ & 30 & $40.00 \%$ & 44 & 44 & 1.938 & 0.164 \\
\hline & gGT & 12 & $48 \%$ & 26 & $34.67 \%$ & 38 & 38 & 1.392 & 0.238 \\
\hline \multirow[t]{2}{*}{ Chi-square } & $\mathbf{X}^{2}$ & & 15.765 & & 10.452 & & & & \\
\hline & P-value & & 0.008 & & 0.063 & & & & \\
\hline
\end{tabular}


Table 4 showed the six virulent factors from the studied samples were as follows: Cag A (65\%). Vac A (53\%). Gro EL (55\%). Urea A (52\%). Hcp c (44\%) and gGT (38\%).

On comparing results of gastric carcinoma and chronic gastritis, it was found that Cag A was detected in 88\% (22/25) by group I. While, it was detected by group II in $57.33 \%$ (43/75) of cases showed statistically significant difference between the 2 studied groups. Vac A was detected in $80 \%$ (20/25) by group I while, it was detected by group II in $44 \%(33 / 75)$ of cases showed statistically significant difference between the 2 studied groups.

Gro EL was detected in $72 \%(18 / 25)$ in group I while, it was $49.33 \%$ (37/75) in group II with statistically significant difference between the 2 studied groups. Urea A was 52\% (13/25) in group I while, in group II it was 52\% (39/75) of cases with no statistically significant difference between the different groups.

Hcp c was 56\% (14/25) in group I while, in group II it was 40\% (30/75) of cases with no statistically significant difference between the 2 studied groups. gGT was detected in $48 \%(12 / 25)$ in group I while, in group II it was $34.67 \%(26 / 75)$ of cases with no statistically significant difference between the different groups. In this study, a statistically significant association was found between Cag A, Vac A and Gro EL antigen in the studied groups of patients. However, no such statistically significant association was found between Urea A, Hcp c and gGT antigen. ( $\mathrm{P}$ value $>0.05)$.

Table 5: relationship between Cag A and sex, smoking and complaining symptoms

\begin{tabular}{|c|c|c|c|c|c|c|c|c|}
\hline & & & & & \multicolumn{2}{|r|}{ Cag A } & \multicolumn{2}{|c|}{ Chi-square } \\
\hline & \multicolumn{2}{|c|}{ Negative Cag A (35) } & \multicolumn{2}{|c|}{ Positive Cag A (65) } & \multicolumn{2}{|r|}{ Total } & & \\
\hline & $\mathbf{N}$ & $\%$ & $\mathbf{N}$ & $\%$ & $\mathbf{N}$ & $\%$ & $\mathbf{X}^{2}$ & P-value \\
\hline Female & 25 & $71.4 \%$ & 35 & $53.8 \%$ & 60 & $60.0 \%$ & 2.999 & 0.083 \\
\hline Male & 10 & $28.6 \%$ & 30 & $46.2 \%$ & 40 & $40.0 \%$ & & \\
\hline Smoker & 7 & $20.0 \%$ & 22 & $33.8 \%$ & 29 & $29.0 \%$ & 2.201 & 0.138 \\
\hline Dyspepsia & 12 & $34.3 \%$ & 8 & $12.3 \%$ & 20 & $20.0 \%$ & 6.585 & $0.010^{*}$ \\
\hline Pain & 17 & $48.6 \%$ & 28 & $43.1 \%$ & 45 & $45.0 \%$ & 0.277 & 0.599 \\
\hline Vomiting & 5 & $14.3 \%$ & 18 & $27.7 \%$ & 23 & $23.0 \%$ & 2.444 & 0.118 \\
\hline hematemesis & 2 & $5.7 \%$ & 1 & $1.5 \%$ & 3 & $3.0 \%$ & 1.283 & 0.257 \\
\hline Dysphagia & 0 & $0.0 \%$ & 6 & $9.2 \%$ & 6 & $6.0 \%$ & 5.374 & $0.020^{*}$ \\
\hline
\end{tabular}

Table 5 showed among the studied groups there were 60 females [25 (71.4\%) of the 35 negative Cag A cases and 35 (53.8) of 65 positive Cag A cases] and 40 males [10 (28.6\%) in 35 negative Cag A and 30 (46.2\%) in 65 positive Cag A cases]. There were 29 smokers $[7$ (20\% of the 35 negative Cag A cases) and 22 (33.8\%) of 65 positive Cag A cases] with no statistically significant difference.

In the present study, 20 were suffered from dyspepsia [8 (12.3\%) of the 35 negative Cag A cases and 12 (34.3\%) of 65 positive Cag A cases] which statistically significant and 45 were suffered from pain [17 (48.6\%) of the 35 negative Cag A cases and 28 (43.1\%) of 65 positive Cag A cases] with statistically non-significant difference. There were 23 suffered from vomiting [ $5(14.3 \%)$ of the 35 negative Cag A cases and $18(27.7 \%)$ of 65 positive Cag A cases] and 3 suffered from hematemesis [2 (5.7\%) of the 35 negative Cag A cases and $1(1.5 \%)$ of 65 positive Cag A cases] with statistically non-significant. Dysphagia were detected in 6 patients in positive Cag A and none of negative Cag A with statistically significant difference.

Table 6: relationship between Cag $A$ and the others antigen

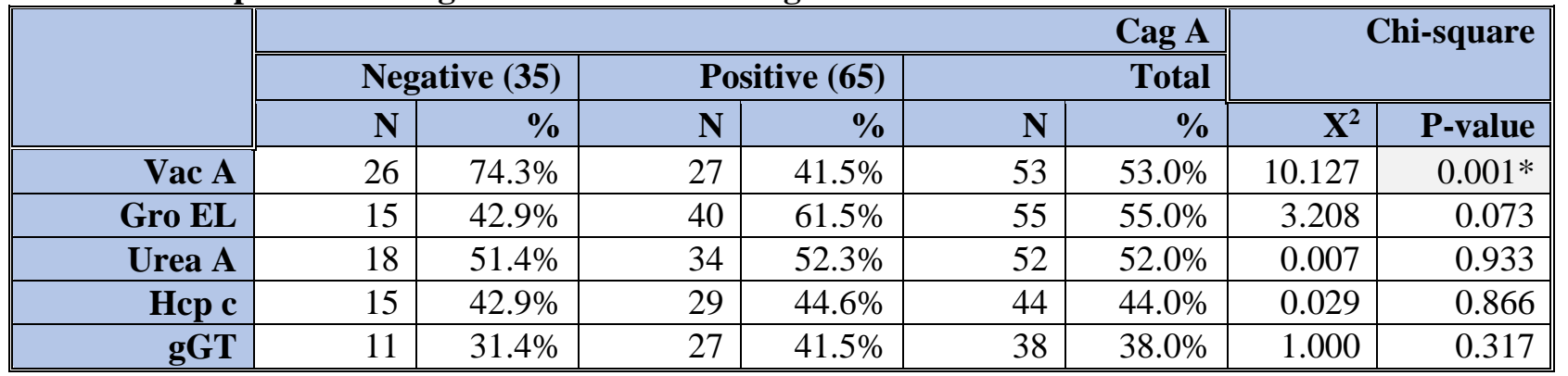

Table 6 showed that we were interested to re-evaluate the association between Cag A antigen and the other five antigens, the prevalence of Vac A antigen was 27 out of 65 positive Cag A (41.5\%) and $26(74 \%)$ out of 35 negative Cag A with statistically significant difference (P. value $<0.05)$. 
Gro El antigen was 40 (61.5\%) positive Cag A out of 65 sample and 15 (42.9\%) out of 35 negative Cag A with no statistically significant, Urea A antigen was 34 (52.3\%) positive Cag A out of 65 sample and 18 (51.4\%) out of 35 negative Cag A with no statistically significant difference.

Hcp c antigen was 34 (52.3\%) positive Cag A out of 65 sample and 15 (42.9\%) out of 35 negative Cag A with no statistically significant, gGT antigen was 27 (41.5\%) positive Cag A out of 65 sample and 11 (31.4\%) out of 35 negative Cag A with no statistically significant difference.

Table 7: relationship between Vac A and sex, smoking and complaining symptoms

\begin{tabular}{|c|c|c|c|c|c|c|c|c|}
\hline & & & & & \multicolumn{2}{|r|}{ Vac A } & \multicolumn{2}{|c|}{ Chi-square } \\
\hline & \multicolumn{2}{|c|}{ Negative Vac A (47) } & \multicolumn{2}{|c|}{ Positive Vac A (53) } & \multicolumn{2}{|r|}{ Total } & \multirow[b]{2}{*}{$\mathbf{X}^{2}$} & \multirow[b]{2}{*}{ P-value } \\
\hline & $\mathbf{N}$ & $\%$ & $\mathbf{N}$ & $\%$ & $\mathbf{N}$ & $\%$ & & \\
\hline Female & 32 & $68.1 \%$ & 28 & $52.8 \%$ & 60 & $60.0 \%$ & \multirow[t]{2}{*}{2.433} & \multirow[t]{2}{*}{0.119} \\
\hline Male & 15 & $31.9 \%$ & 25 & $47.2 \%$ & 40 & $40.0 \%$ & & \\
\hline Smoker & 11 & $23.4 \%$ & 18 & $34.0 \%$ & 29 & $29.0 \%$ & 1.360 & 0.243 \\
\hline Dyspepsia & 7 & $14.9 \%$ & 13 & $24.5 \%$ & 20 & $20.0 \%$ & 1.468 & 0.226 \\
\hline Pain & 24 & $51.1 \%$ & 21 & $39.6 \%$ & 45 & $45.0 \%$ & 1.319 & 0.251 \\
\hline Vomiting & 11 & $23.4 \%$ & 12 & $22.6 \%$ & 23 & $23.0 \%$ & 0.008 & 0.928 \\
\hline hematemesis & 1 & $2.1 \%$ & 2 & $3.8 \%$ & 3 & $3.0 \%$ & 0.237 & 0.626 \\
\hline Dysphagia & 1 & $2.1 \%$ & 5 & $9.4 \%$ & 6 & $6.0 \%$ & 2.593 & 0.107 \\
\hline
\end{tabular}

Table 7 showed regarding the relationship between positive Vac A antigen with female and male, there were frequently 28 females out of 53 positive Vac A (52.8\%) and 25 males out of 53 positive Vac A (47.2\%) with no statistically significant difference. Concerning the smoking habits of the studied groups, there was no statistically significant difference between positive Vac A antigen 18 (34\%) and negative Vac A 11(23.4\%). As regard the relationship between Vac A and complaining symptoms there was no statistically significant difference. Positive 53 Vac A cases was obtained in dyspepsia 13 (24.5\%), pain 21 (39.6\%), vomiting 12 (22.6\%), hematemesis $2(3.8 \%)$ and dysphagia $5(9.4 \%)$.

Table 8: relationship between Vac $A$ and the other antigen

\begin{tabular}{|c|c|c|c|c|c|c|c|c|}
\hline & & & & & \multicolumn{2}{|r|}{ Vac A } & \multicolumn{2}{|c|}{ Chi-square } \\
\hline & \multicolumn{2}{|c|}{ Negative (47) } & \multicolumn{2}{|c|}{ Positive (53) } & \multicolumn{2}{|r|}{ Total } & & \\
\hline & $\mathbf{N}$ & $\%$ & $\mathbf{N}$ & $\%$ & $\mathbf{N}$ & $\%$ & $\overline{X^{2}}$ & P-value \\
\hline Gro EL & 30 & $63.8 \%$ & 25 & $47.2 \%$ & 55 & $55.0 \%$ & 2.811 & 0.094 \\
\hline Urea $A$ & 29 & $61.7 \%$ & 23 & $43.4 \%$ & 52 & $52.0 \%$ & 3.366 & 0.067 \\
\hline Нср с & 20 & $42.6 \%$ & 24 & $45.3 \%$ & 44 & $44.0 \%$ & 0.075 & 0.784 \\
\hline gGT & 12 & $25.5 \%$ & 26 & $49.1 \%$ & 38 & $38.0 \%$ & 5.956 & $0.015^{*}$ \\
\hline
\end{tabular}

Table 8 showed that Gro EL was detected in $47.2 \%$ (25/53) by positive Vac A. While it was detected by negative Vac A in $63.8 \%$ (30/47). Urea A was detected in $43.4 \%$ (23/53) by positive Vac A while it was detected by negative Vac A in $61.7 \%$ (29/47) of cases with no statistically significant difference.

Hcp c was detected in $45.3 \%$ (24/53) by positive Vac A, while it was detected by negative Vac A in 42.6\% (20/47) with no statistically significant difference. gGT was detected in $43.4 \%(26 / 53)$ by positive Vac A while it was detected by negative Vac A in $25.5 \%$ (12/47) of cases with statistically significant difference. 


\section{DISCUSSION}

Several tests are available for the detection of $\mathrm{H}$. pylori. However, because of the high prevalence of infection, only a few tests are applicable for population-based screening approaches. First, the tests must be easy to handle and cheap to perform. Furthermore, they should have high sensitivity and specificity ${ }^{[8]}$. In this study, our aim was to investigate the seroprevalence of six highly immunogenic virulence factors (Cag A, Vac A, Gro EL, gGT, Hcp $\mathrm{C}$, and Ure A) in patients with different gastric histology in a high-risk population of gastric cancer and explores the relationship between $H$. pylori virulence factors and gastric carcinoma.

In the present study, $65 \%$ of $H$. pylori were positive for the cag A. Comparable results were represented by Watada et al. ${ }^{[9]}$, Joutei et al. ${ }^{[10]}$ and Salih et al. ${ }^{[11]}$ where cag A positive strains accounted for $25.5 \%, 29 \%$ and $26.4 \%$, respectively. Against that Datta et al. ${ }^{[12]}$ reported cag A positive strains was $100 \%$ in India.In the present study, Cag A was detected in $88 \%(22 / 25)$ in group I. While, in group II it was $57.33 \%(43 / 75)$ with statistically significant difference between the 2 studied groups.

A study had suggested that cag A is a useful marker for the most virulent strains that are associated with gastritis and gastric cancer ${ }^{[13]}$. When comparing the prevalence of the cag A between the GC and gastritis groups, a highly significant statistical difference was noticed in favor of the GC patients; $16 / 30(53.3 \%)$ vs $4 / 30(13.3 \%)$ for the gastritis patients ( $\mathrm{P}$ value of 0.001).

Our results are different from those reported by Meira et al. ${ }^{[8]}$ who found that the association between gastric cancer and sero-positivity to the Cag A, did not reach statistical significance in the study for the entire population. Cag A was detected in 91.6\% (208/227) in group I. While, in group II it was $88.7 \%$ (400/451) of cases.In the present study, Vac A was detected in $80 \%(20 / 25)$ in group I while; in group II it was $44 \%$ (33/75) with statistically significant difference between the studied groups.

Our results agree with those of Figura et al. ${ }^{[14]}$ who confirmed that clinical diagnosis shows that 738 patients were with chronic gastritis, 260 with peptic ulcer disease (119 with gastric ulcer, 133 duodenal ulcers and 8 gastric and duodenal ulcers) and 81 patients were with gastric cancer (GC). Vac A was present in $112(15.2 \%)$ of patients with chronic gastritis, $24(20.1 \%)$ of patients with gastric ulcer, 35 $(26.3 \%)$ of patients with duodenal ulcer and 44 $(54.3 \%)$ of patient with gastric cancer ( $\mathrm{p}<0.001)$. Our findings are different from those reported by Meira $\boldsymbol{e} t$ al. ${ }^{[8]}$ in Shanghai among Chinese men whom found the association between gastric cancer and sero- positivity to the Vac A, did not reach statistical significance in the study. Vac A was detected in $88.9 \%$ ( $201 / 227)$ by gastric carcinoma, while it was detected by gastritis in $83.8 \%$ (378/451) of cases.In our study, Gro EL was detected in 72\% (18/25) in group I while, in group II it was $49.33 \%$ (37/75) of cases with statistically significant difference between the 2 studied groups. Our results agree with those of Gao et al. ${ }^{[15]}$ in Germany, who evaluated H. pylori which included 123 gastric cancer cases and 492 controls cases. Seropositivities for Cag A and Gro EL were identified as independent risk predictors, which were strongly related to $\mathrm{GC}$ risk in a dose-response manner. Seropositive of the screening Gro EL 280/492 (56.9\%) was observed in controls, but a much higher positivity for Gro EL 103/123 (83.7\%) was seen in GC cases showing statistically significant difference between the 2 studied groups.

Our results are different from those detected by Meira et al. ${ }^{[8]}$ in Shanghai among Chinese men whom found Gro EL did not reach statistically significant difference between gastric cancer and gastritis. GroEL was detected in $86.3 \%(195 / 227)$ by gastric cancer cases, while it was detected by gastritis cases in 85.1 $\%$ (384 /451).In the present study, three highly immunogenic virulence factors (gGT, Hcp C, and Ure A) were detected in cases with no statistically significant difference between the gastric carcinoma and gastritis groups.

Urea A was detected in 52\% (13/25) in group I while in group II it was 52\% (39/75). Hcp c was detected in 56\% (14/25) in group I while in group II it was $40 \%(30 / 75)$ of cases. gGT was detected in $48 \%$ $(12 / 25)$ in group I while in group II it was $34.67 \%$ $(26 / 75)$ of cases.

Our results are in agreement with those of Gao et al. ${ }^{[15]}$ who showed that Among the 2 studied groups, there were $89 \mathrm{Hcp} \mathrm{C}$ gastric carcinoma seropositive (72.4\%) and 270 Hcp C gastritis seropositive $(54.9 \%)$. Seropositivity of the screening Ure A 42 (34.1\%) was observed in gastric carcinoma, and a positivity for Ure A 195 (39.6\%) was seen in gastritis cases.

Our results are like those reported by Meira $\boldsymbol{e t}$ al. ${ }^{[8]}$, who found sero-positive of Ure A was 116 gastric carcinoma cases $(51.3 \%)$ and 236 gastritis cases $(52.3 \%)$, Sero-positive of Hcp c was 134 gastric carcinoma cases $(59.3 \%)$ and 266 gastritis case (59\%) with no statistically significant difference between the 2 studied groups.

In this study, we were interested to re-evaluate the association between Cag A antigen and the other five antigens, the prevalence of Vac A antigen was 27 out of 65 positive Cag A (41.5\%) and 26 (74\%) out of 
35 negative Cag A with statistically significant difference $(P$. value $<0.05)$.

In other study, in accordance with ours, Mounia et al. ${ }^{[16]}$ reported the correlation between cag $\mathrm{A}$ and vac $\mathrm{A}$ genotypes. The results show a significant association between vac A and positive cag A (45.4\%) and between Vac A and negative Cag A (78.6\%). Our results are different from those found by Pinto et al. ${ }^{[17]}$ in China who studied the H. pylori Vac A and Cag A-positive strains, were found with similar frequencies in patients with chronic gastritis and patients with gastric carcinoma, and consequently, no significant relationships could be disclosed between Vac A or Cag A genotypes. The results show a nonsignificant association between $\mathrm{Vac} A$ and negative CagA (39.4\%) and between Vac A and positive Cag A $(37.6 \%)$.

\section{CONCLUSION}

The newly developed test (RecomLine) is a line immunoassay based on recombinantly expressed $H$. pylori proteins. In contrast to ELISA, the test principle allows the identification of specific antibodies against various antigens of $H$. pylori through the separate application of different single antigens. In this study, a statistically significant association was found between Cag A, Vac A and Gro EL antigen in the studied groups of patients. However, no such statistically significant association was found between Urea A, Hcp c and gGT antigen. (P value > 0.05).

\section{REFERENCES}

1. Alaoui S, Amarti A, El Rhazi $\mathrm{K}$ et al. (2013): Helicobacter pylori genotypes associated with gastric histo-pathological damages in a Moroccan population. PloS One, 8(12):243-257.

2. Malfertheiner P, Megraud F, O'Morain C et al. (2012): Management of Helicobacter pylori infection. Florence Consensus Report. Gut, 61:646-664.

3. Shiota S, Murakami K, Fujioka T et al. (2010): Population-based strategies for Helicobacter pyloriassociated disease management: a Japanese perspective. Expert Rev. Gastroenterol. Hepatol., 4:149 156.

4. Hatakeyama $M$ and Higashi $H$ (2005): Helicobacter pylori Cag A: a new paradigm for bacterial carcinogenesis. Cancer Sci., 96:835-843.
5. Haley K and Gaddy J (2015): Helicobacter pylori: genomic insight into the host-pathogen interaction. Int. J. Genomics, 15: 505-509.

6. Miftahussurur M, Yamaoka Y, Graham D et al. (2017): Helicobacter pylori vacA as an oncogenic pathogen, revisited. Expert. Rev. Mol. Med.,19:133-139.

7. Rahimian G, Sanei M, Shirzad H et al. (2014): Virulence factors of Helicobacter pylori increase markedly gastric mucosal TGF-beta1 mRNA expression in gastritis patients. Microb. Pathog., 7:67-68.

8. Meira E, Wei Z, Yong-Bing X et al. (2012): Prospective study of Helicobacter pylori biomarkers for gastric cancer risk among Chinese men Cancer Epidemiol. Biomarkers Prev., 21(12): 2185-2192.

9. Watada M, Shiota S, Matsunari O et al. (2011): Association between Helicobacter pylori cagA-related genes and clinical outcomes in Colombia and Japan. BMC.Gastroenterol., 11:141-143.

10. Joutei H, Hilali A, Fechtali $\mathbf{T}$ et al. (2010): Helicobacter pylori infection in 755 patients with digestive complaints. East. Mediterr. Health J., 16(7):778782 .

11. Salih B, Bolek B, Arikan S et al.(2010): DNA sequence analysis of cagA $3^{\prime}$ motifs of Helicobacter pylori strains from patients with peptic ulcer diseases. J. Med. Microbiol., 59(2):144-148.

12. Datta S, Chattopadhyay S, Balakrish G et al. (2003): Virulence genes and neutral DNA markers of Helicobacter pylori isolates from different ethnic communities of West Bengal. India J. Clin. Microbiol., 41(8):3737-3743.

13. Tanih N, McMillan $M$, Naidoo $N$ et al. (2010): Prevalence of Helicobacter pylori vacA,cagA and iceA genotypes in South African patients with upper gastrointestinal diseases. Acta Trop., 116:68-73.

14. Figura N, Valassina $M$, Moretti E et al.(2015): Histological variety of gastric carcinoma and Helicobacter pylori Cag A and Vac A polymorphism. Eur. J. Gastroenterol. Hepatol., 27(9):1017-1021.

15. Gao L, Michel A, Weck MN et al. (2009): Helicobacter pylori infection and gastric cancer risk: evaluation of $15 \mathrm{H}$. pylori proteins determined by novel multiplex serology. Cancer Res., 69:6164-6170.

16. Mounia E, Samia A, Dafr-Allah B et al. (2017): Vac A and Cag A status as biomarker of two opposite end outcomes of Helicobacter pylori infection (Gastric cancer and duodenal ulcer) in a Moroccan population. PLoS One, 12(1):32-39.

17. Pinto-Ribeiro I, Ferreira R, Batalha $S$ et al. (2016): Helicobacter pylori vacA genotypes in chronic gastritis and gastric carcinoma patients. Macau China, 8(5):153-159. 